DRAFT VERSION SEPTEMBER 25, 2018

Preprint typeset using LTEX style emulateapj v. 5/2/11

\title{
ON THE SIZE AND LOCATION OF THE X-RAY EMITTING CORONAE AROUND BLACK HOLES
}

\author{
R. C. REIS ${ }^{1,2}$, J. M. MILleR ${ }^{1}$, \\ Draft version September 25, 2018
}

\begin{abstract}
The observation of energetic X-ray emission from black holes, inconsistent with thermal emission from an accretion disk, has long indicated the presence of a "corona" around these objects. However, our knowledge of the geometry, composition, and processes within black hole coronae is severely lacking. Basic questions regarding their size and location are still a topic of debate. In this letter, we show that for black holes with luminosities $L \gtrsim 10^{-2} L_{E d d}$ - characteristic of many Seyferts, quasars, and stellar-mass black holes (in their brighter states) - advanced imaging and timing data strongly favor X-ray emitting regions that are highly compact, and only a few Gravitational radii above the accretion disk. The inclusion of a large number of possible systematics uncertainties does not significantly change this conclusion with our results still suggesting emission from within $\sim 20 r_{\mathrm{g}}$ in all cases. This result favors coronal models wherein most of the hard X-ray emission derives from magnetic reconnection in the innermost disk and/or from processes in the compact base of a central, relativistic jet.
\end{abstract}

\section{INTRODUCTION}

Observations of accreting black holes (BHs) often indicate the presence of hard X-rays having energies much greater than the expected thermal peak of the accretion disk. This is true not only for Active Galactic Nuclei (AGNs; e.g. Elvis et al. 1978 ) but also for X-ray binaries (XRBs; e.g. White \& Holt 1982).

It has been widely postulated that hard X-rays are the product of inverse Compton scattering of seed photons from accretion disks by hot "coronae" (e.g. Haardt \& Maraschi 1991), however despite our observational (e.g. Zdziarski et al. 1999) and theoretical (e.g. Schnittman et al. 2012, and references therein) efforts, there are still a number of fundamental questions regarding the geometry, or composition of accretiondisk coronae.

At low luminosities $\left(L \lesssim 10^{-4} L_{E d d}\right.$, where $L_{E d d}$ is the Eddington luminosity), the hard-X-rays are thought to originate from a hot, quasi-spherical and large $\left(\sim 100 \mathrm{GM} / \mathrm{c}^{2} \sim\right.$ $100 r_{\mathrm{g}}$ ) advection-dominated accretion flow (ADAF) which replaces the innermost disk around the $\mathrm{BH}$ (e.g. Narayan \& Yi 1994). On the other hand, at luminosities $L \gtrsim 10^{-2} L_{E d d}$, observations of XRBs and Seyfert AGNs show the clear presence of a more compact X-ray emitting source (the corona; e.g. McHardy et al. 2006) on top of a geometrically-thin, optically thick accretion disk extending as far in as the radius of the innermost stable circular orbit (ISCO).

Amongst the clearest evidence for the co-existence of the corona with the disk is the presence of Fe K-shell emission lines together with other reflection features in the X-ray spectra of these sources (Miller 2007, and references therein). From the profile of these emission lines we are able to obtain information on the effects of strong Doppler and gravitational redshifts natural to the space-time around $\mathrm{BHs}$ and measure their spin (see Miller 2007, for a review).

Having a tool to probe the innermost regions around $\mathrm{BHs}$ and to measure their spin $\left(a=c J / G M^{2},-1<a<1\right)$ is of particular importance as the distribution of spin in nearby supermassive black holes (SMBHs) can be used to distinguish

\footnotetext{
${ }^{1}$ Dept. of Astronomy, University of Michigan, Ann Arbor, Michigan 48109, USA

${ }^{2}$ Einstein Fellow
}

between various $\mathrm{BH}$ growth scenarios (e.g. Berti \& Volonteri 2008), and in the case of stellar mass BHs, their spin distribution relates to the distribution of angular momentum of the progenitors (e.g. Miller et al. 2011). Knowledge of spin is also essential if one wishes to test if relativistic jets can be powered by the spin energy of BHs via the Blandford-Znajek process (e.g. Narayan \& McClintock 2012) or, in the near future, if there is evidence for violations of the No-Hair Theorem (e.g. Johannsen \& Psaltis 2011).

The location and compactness of the corona - which is irradiating the disk and giving rise to the reflection features - are thus of paramount importance in the study of strong gravity. Nonetheless, in theoretical consideration of the corona it is often assumed to be compact and located in the inner regions, $\lesssim 10 r_{\mathrm{g}}$ around the $\mathrm{BH}$ (e.g. Miniutti \& Fabian 2004, and references therein). This assumption has consistently been successful in describing the spectral and timing properties of BHs at the highest signal-to-noise available, however strong deviation from this could affect our ability to probe strong gravity around BHs (Dauser et al. 2013).

Furthermore, coronae are known to play an important role in state transitions in XRBs (e.g. Reis et al. 2013, and references therein); and jets in both XRBs and AGNs have often been strongly linked with the corona (e.g. Merloni et al. 2003 ). If the corona is the base of such a jet (Falcke \& Biermann 1999), then the amount and the form in which energy escapes through the jet is largely dependent on the enigmatic properties of the corona, highlighting the importance in establishing the size and location of these regions.

In this letter, we explore the results aimed at measuring the physical sizes and locations of coronae around luminous accreting SMBHs $\left(L \gtrsim 10^{-2} L_{E d d}\right.$ typical of bright nearby Seyferts and quasars). We find that in all cases the coronae are located significantly closer than $\sim 10 r_{\mathrm{g}}$ from the accretion disks and their sizes are suggestive of being highly compact. These results give credence to the assumptions often employed in most reflection-based studies of BHBs and AGNs.

\section{LOCATION BASED ON REVERBERATION LAGS}

With the discovery of a soft X-ray reverberation lag of $\sim 30$ s in the AGN 1H 0707-495 (Fabian et al. 2009), a new method to measure the distance $\left(D_{\text {cor }}\right)$ between the X-ray 

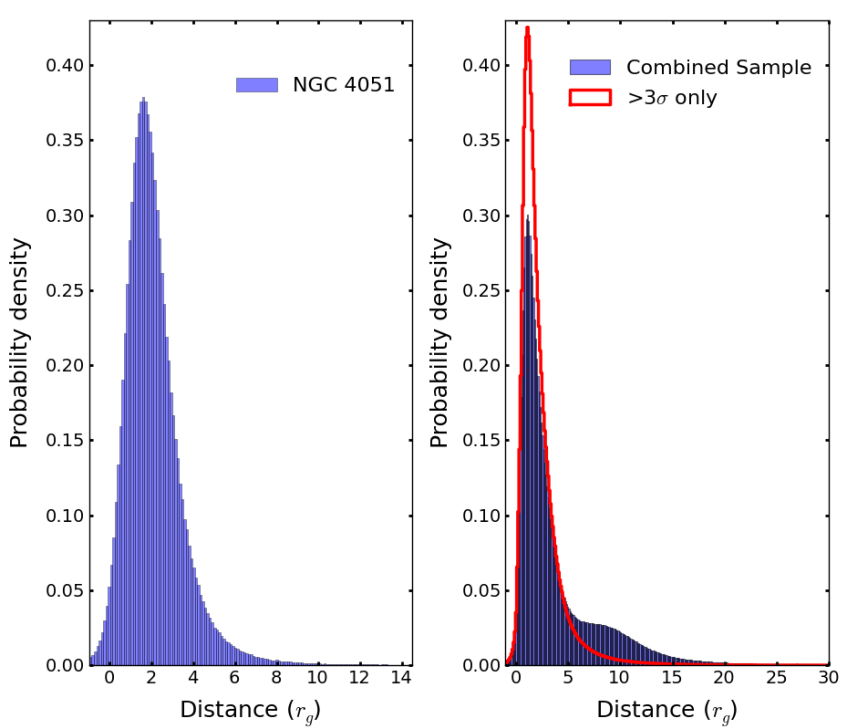

FIG. 1.- Left: Representative probability distribution for the distance between the accretion disk and the corona $\left(D_{\text {cor }}\right)$ based on soft X-ray reverberation lags. Right: Probability distribution for the combined sample of 17 Seyferts (blue). The tail extending to $\sim 15 r_{\text {g }}$ comprises of RE J1034+396, NGC 4151 and ESO 113-G010. Shown in red is the distribution for sources with lag detection at $>3 \sigma$, with the exception of NGC 4151 .

emitting region and the accretion disks was established. The decomposition of the spectrum of 1H 0707-495 into direct and reflected emission components supported interpreting the observed lag as a signature of the light travel time between the corona (the hard X-ray continuum source) and the accretion disk (which reprocess the flux into a reflection component with atomic spectral features).

Despite the success that this "reflection" model had in explaining the timing and spectral features of $1 \mathrm{H} \mathrm{0707-495}$ (Fabian et al. 2009), a further interpretation was soon made available where the lag was due to the reverberation caused by scattering of X-rays passing through an absorbing medium that happen to lie in the line of sight to the central AGN (Miller et al. 2010). However, the subsequent discovery of similar lags in a large number of sources (see below), spanning a large range of masses, Eddington fractions, and inclination angles, is likely incompatible with the specialized partial covering geometry required to explain the lags. Thus, the original light-crossing argument remains the best interpretation for these soft lags. For a detailed description of soft Xray reverberation techniques and its applications see Zoghbi et al. (2012); Wilkins \& Fabian (2013).

At the time of writing, there were a total of 17 radio-quiet Seyfert ${ }^{3}$ with measured soft lags that met our luminosity criteria. This sample is shown in Table 1 together with estimates for the mass and luminosity as a fraction of Eddington to each source collected from the literature. The lags compiled here are taken from the work of De Marco et al. (2012); Zoghbi et al. (2012); Fabian et al. (2012); Cackett et al. (2012) and Kara et al. (2013), whereas the masses are primarily from Zhou et al. (2010); Zhou \& Wang (2005); Bentz et al. (2006) and Onken et al. (2007). As Seyferts are known to be highly variable, the luminosities shown here are meant to be seen

${ }^{3}$ The LLAGN NGC 4395 is not used in this work as it accretes constantly at $L / L_{E d d} \sim 1.2 \times 10^{-3}$ and is possibly already in the ADAF regime and thus intrinsically different from the more luminous systems.

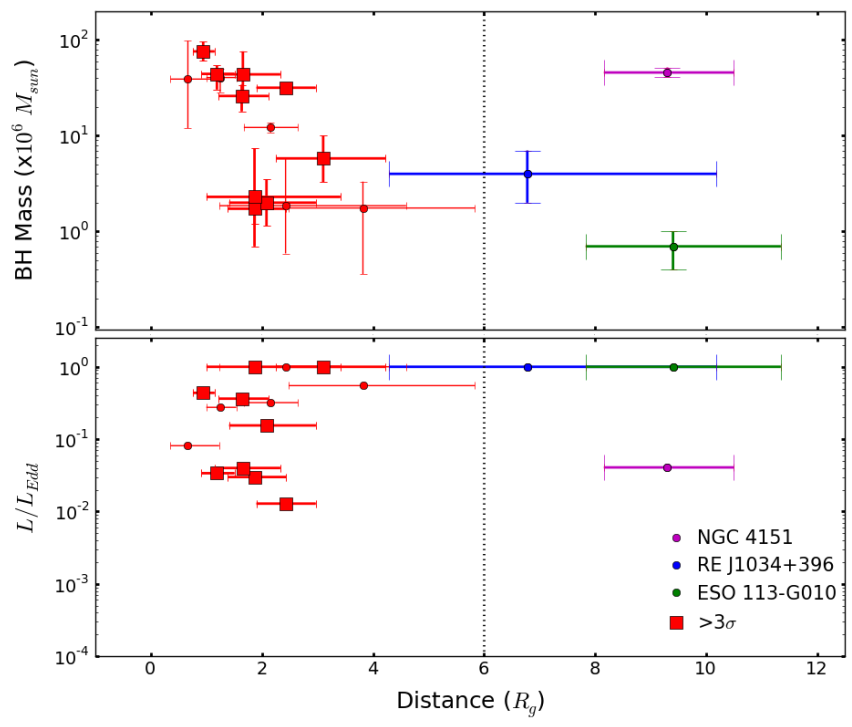

FIG. 2.- BH mass (top) and $L / L_{E d d}$ (bottom) as a function of disk-corona distance. The dashed line marks the ISCO of a non-rotating BH $\left(6 r_{\mathrm{g}}\right) .14 / 17$ sources in our sample suggest a corona close to the accretion disk. These results are independent of the mass or $\dot{m}$ as long as the system are accreting above a few percent of Eddington. The three singled out sources corresponds to those peaking at $\sim 9 r_{\mathrm{g}}(\S 2)$ and the sources with lag detection at $>3 \sigma$ level are shown with square markers.

only as a rough indication of the average luminosity of each system.

Due to the scale-invariant nature of the physics governing accretion flows onto BHs, the lag time scale $t$ depend primarily on the mass of the central $\mathrm{BH}^{4} t \sim G M / c^{3}=r_{g} / c$. We estimate the probability distribution for the distances between the coronae and accretion disks by drawing samples from the probability distribution of the lag times and masses as obtained from the literature and reported in Table 1. For NGC 4051, MRK 766, RE'J1034+396, NGC 7469, Mrk 335, PG 1211+143, NGC 3516, NGC 5548 and NGC 4151, their masses were assumed to have a Gaussian distribution with standard deviation given by the average of the positive and negative errors shown in Table 1, when the reported errors were asymmetric. For the remaining sources with the exception of ESO-113-G010, the logarithm of the masses were assumed to have a Gaussian distribution with the symmetric errors reported in each reference. Finally, for ESO-113-G010, we assumed a uniform distribution in the range given in Table 1.

An example of a derived probability distribution based on $10^{6}$ samples is shown in Fig. 1 (left) for NGC 4051. We report on the 5th column of Table 1 the median of $D_{\text {cor }}$ with the $1 \sigma$ error taken to be the standard deviation of each source sample. Figure 1 (right) shows the distribution for the combined sample, where it becomes clear that there is a peaks at $\sim 2 r_{\mathrm{g}}$ and a further, minor peak at $\sim 9 r_{\mathrm{g}}$ with a tail that extends up to $\sim 15 r_{\mathrm{g}}$.

The 3 sources giving rise to the second peak at larger $D_{\text {cor }}$ are NGC 4151, RE J1034+396 and ESO 113-G010. There is currently uncertainty on the mass of the SMBH in

\footnotetext{
${ }^{4}$ This simple formula does not take into account the effect of the Shapiro time delay; however, this correction is of the order of $0.5 r_{\mathrm{g}}$. The apparent distance between the corona and reflector, calculated using the simplified formula and neglecting the Shapiro delay, is therefore larger than the actual distance (Wilkins \& Fabian|2013 for details).
} 
Table 1: Summary of sources used in this work. The table is divided into two with the top half referring to the 17 Seyferts and the bottom half to the 5 microlensed quasars. The various columns are (1) Object name; (2) black hole masses in Solar units. For the quasars, their masses are in logarithmic scale as quoted in the literature; (3) representative Eddington fraction; (4) lag time in seconds (top) or logarithmic of the size of the X-ray emitting region in centimetres (bottom); (5) The median of the distance, $\left\langle D_{\text {cor }}\right\rangle$, between the corona and the accretion disk in gravitational units (top) or the median of the size of the corona $\langle S\rangle$ also in gravitational units (bottom).

\begin{tabular}{|c|c|c|c|c|}
\hline $\begin{array}{l}\text { Object } \\
\text { (1) }\end{array}$ & $\begin{array}{c}\text { Mass }\left(\times 10^{6} \mathrm{M}_{\odot}\right) \\
(2)\end{array}$ & $\begin{array}{c}L / L_{E d d} \\
\text { (3) }\end{array}$ & $\begin{array}{c}\text { Soft Lag Time (s) } \\
\text { (4) }\end{array}$ & $\begin{array}{c}D_{\text {cor }}>\left(r_{\mathrm{g}}\right) \\
(5)\end{array}$ \\
\hline NGC 4051 & $1.73_{-0.52}^{+0.55}$ & 0.03 & $16 \pm 8$ & $1.9_{-0.5}^{+0.6}$ \\
\hline Mrk 766 & $1.76_{-1.40}^{+1.56}$ & 0.55 & $43 \pm 18$ & $3.8_{-1.3}^{+2.0}$ \\
\hline Ark 564 & $1.86_{-1.27}^{+4.03}$ & 1 & $26 \pm 15$ & $2.4_{-1.2}^{+2.22}$ \\
\hline MCG-6-30-15 & $2.0_{-0.9}^{+1.5}$ & 0.15 & $22 \pm 11$ & $2.1_{-0.7}^{+0.9}$ \\
\hline 1H 0707-495 & $2.3_{-1.6}^{+5.1}$ & 1 & $24 \pm 11$ & $1.9_{-0.9}^{+0.5}$ \\
\hline RE J1034+396 & $4_{-2}^{+3.6}$ & 1 & $150 \pm 90$ & $6.8_{-2.5}^{+3.4}$ \\
\hline NGC 7469 & $12.2 \pm 1.4$ & 0.32 & $130 \pm 60$ & $2.2 \pm 0.5$ \\
\hline Mrk 335 & $26 \pm 8$ & 0.36 & $210 \pm 100$ & $1.6_{-0.4}^{+0.5}$ \\
\hline PG $1211+143$ & $40.5_{-12.1}^{+9.6}$ & 0.28 & $250 \pm 90$ & $1.3_{-0.2}^{+0.3}$ \\
\hline NGC 3516 & $31.7_{-4.2}^{+2.8}$ & 0.013 & $380 \pm 170$ & $2.4 \pm 0.5$ \\
\hline Mrk 841 & $75.9_{-15.6}^{+19.6}$ & 0.44 & $360 \pm 130$ & $0.9 \pm 0.2$ \\
\hline NGC 5548 & $44.2_{-13.8}^{+9.9}$ & 0.034 & $260 \pm 115$ & $1.2 \pm 0.3$ \\
\hline NCC 6860 & $39_{-27}^{+59}$ & 0.08 & $145 \pm 72$ & $0.7_{-0.3}^{+0.6}$ \\
\hline Mrk 1040 & $43.7_{-18.5}^{+32.2}$ & 0.04 & $380 \pm 170$ & $1.7_{-0.5}^{+0.7}$ \\
\hline NGC 4151 & $45.7_{-4.7}^{+5.7}$ & 0.04 & $2100 \pm 500$ & $9.3 \pm 1.2$ \\
\hline IRAS $13224-3809$ & $5.8_{-2.5}^{+4.2}$ & 1 & $92 \pm 31$ & $3.1_{-0.8}^{+1.1}$ \\
\hline ESO 113-G010 & $4-10$ & 1 & $325 \pm 89$ & $9.4_{-1.6}^{+1.9}$ \\
\hline $\begin{array}{l}\text { Object } \\
\text { (1) }\end{array}$ & $\begin{array}{c}\log \left(M_{B H} / \mathrm{M}_{\odot}\right) \\
(2)\end{array}$ & $\begin{array}{l}L / L_{E d d} \\
\quad(3)\end{array}$ & $\begin{array}{c}\log (\text { Size } / \mathrm{cm}) \\
(4)\end{array}$ & $<\underset{(5)}{S>\left(r_{\mathrm{g}}\right)}$ \\
\hline Q $2237+0305$ & $8.68 \pm 0.36$ & 0.44 & $15.46_{-0.29}^{+0.34}$ & $41_{-17}^{+28}$ \\
\hline RX J1131-1231 & $8.32 \pm 0.62$ & 0.03 & $14.04-14.68$ & $7.4_{-3.8}^{+7.6}$ \\
\hline Q J0158-4325 & $8.2 \pm 0.2$ & 0.4 & $14.3_{-0.5}^{+0.4}$ & $8.5_{-3.5}^{+6.1}$ \\
\hline HE 1104-1805 & $9.37 \pm 0.33$ & 0.36 & $14.2-15.0$ & $1.1_{-0.4}^{+0.7}$ \\
\hline HE 0435-1223 & $8.76 \pm 0.44$ & 0.11 & $<15.07$ & $13.8_{-5.3}^{+8.6}$ \\
\hline PG $1115+080$ & $9.1 \pm 0.2$ & 0.37 & $15.6_{-0.9}^{+0.6}$ & $21_{-12}^{+29}$ \\
\hline
\end{tabular}

NGC 4151. The mass determined from reverberation mapping is $4.57_{-0.47}^{+0.57} \times 10^{7} \mathrm{M}_{\odot}$ (Bentz et al. 2006). A stellar dynamical mass measurement obtained using ground based I-band long-slit spectra and the Schwarzschild orbit superposition code of Valluri et al. (2004) gave only an upper limit of $\sim 4 \times 10^{7} \mathrm{M}_{\odot}$ (Onken et al. 2007). However, a recent result based on high resolution near infrared integral field spectroscopic observations of the CO-band head with the Gemini North NIFS spectrograph, gives a revised stellar dynamical mass of $(8.5 \pm 3) \times 10^{7} \mathrm{M}_{\odot}(\mathrm{M}$. Valluri; private communication) - nearly a factor of 2 greater than previous estimates. If this turns out to be right, $D_{\text {cor }}$ for this source presented in Fig 2 should be closer to $5 r_{\mathrm{g}}$.

As for the other two sources peaking at $\sim 9 r_{\mathrm{g}}$, it is possible that they were indeed caught at a time when their coronae were further than the median. It is interesting that RE $\mathrm{J} 1034+396$ is the only persistent AGN to ever show a quasiperiodic oscillation (QPO; Gierliński et al. 2008). In the stellar-mass BH XTE J1650-500, it has recently been shown that the onset of the more coherent QPOs are associated with the collapse of a corona from $\sim 10 r_{\mathrm{g}}$ during the transition from the hard-intermediate to the soft-intermediate state (Reis et al.2013) 5

\footnotetext{
5 The only other QPO detected from a SMBH comes from a tidal disruption source (Reis et al. 2012) and is not persistently active.
}

\section{SIZE BASED ON MICROLENSING}

The possibility of using the "lens-like" effect of stars on distant object is not new. However, its practical use in directly probing the inner regions around SMBHs was not established until the discovery of a flux-ratio anomaly in optical macroimages of the gravitationally lensed quasar QSO 2237+0305 where the optical flux ratio between the brightest macroimages was statistically inconsistent with unity (Irwin et al. 1989 6. Although originally microlensing was not the only explanation for the flux anomaly problem in the optical (e.g. Dalal \& Kochanek 2002), the systematic detection of an increase in the anomaly magnitude at higher energies strongly favoured microlensing as the cause of these anomalies (e.g. Pooley et al. 2007). As the effect of microlensing increases as the size scale of the lensed region decreases - as long as the source is smaller than the projection of the Einstein radius of the microlens into the plane (for a detailed review of microlensing techniques see Wambsganss 2006) - this discovery confirmed the expected hierarchy in the characteristic sizes of the optical, UV and X-ray emission regions, with the latter often thought to be the most compact.

Microlensing studies at optical/IR have often arrived at accretion disk sizes in the order of $10^{4} r_{\mathrm{g}}$ (e.g. Blackburne et al.

\footnotetext{
${ }^{6}$ Under a smooth gravitational potential, without the effect of microlensing, the brightest image-pairs are expected to be mirror-images of each other and have similar intensities (Metcalf \& Zhao 2002).
} 


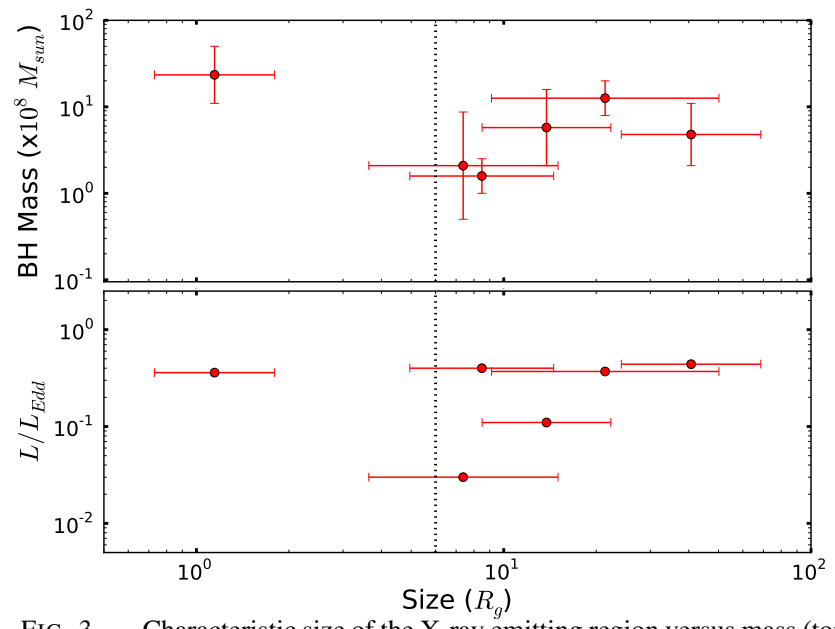

FIG. 3.- Characteristic size of the X-ray emitting region versus mass (top) and characteristic luminosity (bottom).

2006), and most remarkably, such studies have shown that this radius scales with $\mathrm{BH}$ mass in a manner consistent with the expectations of thin-disk theory (Morgan et al. 2010). We are now at a stage where the size of the X-ray emitting corona in the innermost regions around these BHs are beginning to be measured (e.g. Morgan et al. 2008, Chartas et al. 2009; Mosquera et al. 2013, and references therein). We list in Table 1 (Bottom) the details of the 6 sources with a quantitatively derived half-light radius for the X-ray emitting region. These values were compiled from the various work listed above and especially from Fig. 8 of Mosquera et al. (2013). As these are all SMBHs, the accretion disk emission peaks in optical/UV, so the size of the X-ray emitting region therefore directly relates with the corona ${ }^{7}$ In compiling the various masses presented in Table 2, we have searched the literature for the latest $\mathrm{BH}$ mass estimates based on the $\operatorname{MgII}(\lambda 2798 \AA)$ massline width relation for all sources but HE 1104-1805, where $\operatorname{CIV}(\lambda 1549 \AA)$ was used instead. The values quoted are from the work of Peng et al. (2006) and Sluse et al. (2012).

In a similar manner to $\S 2$, we have estimated a probability distribution for the size of the X-ray emitting region of each source in units of $r_{\mathrm{g}}$ based on $10^{6}$ randomly sampled values of mass and size (in $\mathrm{cm}$ ) assuming either a Gaussian distribution with symmetric errors in logarithmic scale or, in the cases where such are not present, a uniform distribution over the range given in Table 1. Figure 3 summarises the result for the characteristic sizes of the coronae.

\section{DISCUSSION}

The two techniques explored in this work effectively probe different aspects of the same emission region. From the reverberation lags, we show in Fig 2 that 14/17 sources in our sample $e^{8}$ are at distances strongly clustered around $2-3 r_{\mathrm{g}}$ above the accretion disk. Taken at face value and assuming that the corona is radially symmetric about the central $\mathrm{BH}$, this immediately suggest that a large fraction of Seyferts should be rapidly spinning (as a point of reference, a radius $R_{\mathrm{ISCO}}=$ $3.15 r_{\mathrm{g}}$ corresponds to a spin parameter $\left.a=0.75 \mathrm{cJ} / G M^{2}\right)$, as is often found with detailed spectral analyses (Walton et al. 2013). For a few sources, notably Mrk 841 and NGC 6860,

\footnotetext{
7 The use of half-light radius in microlensing studies is of particular importance as these have been shown to be independent of the shape or the surface brightness profile of the source (e.g. Mortonson et al. 2005 for details).

8 15/17 using the higher mass estimate of NGC 4151 .
}

the coronae appear to be at distances commensurate with radii for which there are no stable orbits, even for a maximally rotating $\mathrm{BH}^{9}$. However, the effect of lag dilution could artificially reduce the intrinsic lag (e.g. Wilkins \& Fabian 2013) thus increasing the distance between the disk and coron ${ }^{10}$ and possible errors in the $\mathrm{BH}$ mass could also change the $\mathrm{ab}$ solute $D_{\text {cor }}$ reported here.

A number of other systematics uncertainties in the derivation of soft-lags, including the unknown geometry of the corona or intrinsic flux variation (e.g. Zoghbi et al. 2012, Kara et al. 2013) could also increase the radius by a factor of a few. Indeed, for the case of NGC 4151, Zoghbi et al. (2012) showed that the lag-time differed by a factor of $\sim 10$ between high and low flux states. The result presented here conservatively shows the upper value $(\sim 2000 \mathrm{~s})$, as found in the low-state of the source. If instead, we use the $\sim 200 \mathrm{~s} \mathrm{lag}$, the distance between the disk and the corona would commensurate with a single gravitational radii. It is clear that for the majority of the Seyferts reported here, the distance between the $\mathrm{X}$-ray emitting region and the inner accretion disk is smaller than $\sim 20 r_{\mathrm{g}}$ even if we allow for an order of magnitude systematic shift in the derived values. The microlensing results further suggest that these coronae are highly compact, with upper limits on their characteristic sizes in the order of 20$30 r_{\mathrm{g}}$.

The objects explored here span a large range in mass and Eddington fraction; however, in all cases our selection criteria $\left(L \gtrsim 10^{-2} L_{E d d}\right)$ was such that the systems are thought to be in states where the accretion disk extends down to the ISCO (Reis et al. 2013, and references therein);. Based on the presence of highly compact coronae, we postulate that the Seyferts and quasars presented here are in spectral states analogous to the hard/intermediate and the soft/intermediate states of stellar mass BHBs.

The compactness of the coronae in these sources strongly constrains models of Comptonisation, with magnetic reconnection/flares (e.g. Merloni \& Fabian 2001) or failed jet models (e.g. Ghisellini et al. 2004) strongly favoured over models which predict larger, extended coronae (e.g. Haardt \& Maraschi 1991). The small distance between the corona and the accretion disk further suggests that models where the energy is dissipated in the innermost regions or possibly even within the plunging regions, are favoured. Such a scenario was presented by Hirose et al. (2004), where the corona is described as a region of smooth magnetic field lines increasing in strength toward small radii and being strongest for maximally rotating BHs. More recently, global MHD simulations were used to show that the vast majority of the coronal emission comes from a relatively small volume of space (Schnittman et al.2012), consistent with observational results presented in this work.

We also note that the base of the jet in M87 has recently been spatially resolved to be $\lesssim 11 r_{\mathrm{g}}$ in size (Doeleman et al. 2012). If this is associated with the coronae - as is the case in some models (e.g. Falcke \& Biermann 1999) - then this too argues for a highly compact corona.

A number of arguments have so far eluded discussion and shall be mentioned here only briefly. First, the spectral fits to

\footnotetext{
${ }^{9}$ Again under the assumption that the corona is radially symmetric about the central $\mathrm{BH}$.

10 Wilkins \& Fabian 2013 , showed that this effect could increase $D_{\text {cor }}$ in $1 \mathrm{H} 0707-495$ by up to $75 \%$, and a factor of similar magnitude could also affect all other lags mentioned here.
} 
broad, relativistic lines profiles often require emissivities that are indicative of a very compact corona. This has been seen in both stellar-mass as well as SMBH and is often interpreted as the gravitational bending of light toward the inner regions of the disk (Miniutti \& Fabian 2004). We have chosen not to use this line of evidence as argument for a compact corona as it has recently been shown that these results could potentially be dependent on the prior assumption that the corona is inherently compact (Dauser et al.|2013).

The presence of compact coronae are also often inferred by the existence of high frequency QPOs whose frequencies are similar to that expected from the Keplerian frequencies in the inner few gravitational radii from the black hole. QPOs are known to be stronger at higher energies which has prompted the association of the corona as the main instrument in modulating the signals. Indeed, as mentioned above, QPOs have been seen in RE J1034+396 and in the tidal disruption source Swift J164449.3+573451 (Reis et al.|2012), and in both cases the QPOs have been associated with the coronae as the emission from the disk peaks in the optical/UV. However, the drawback in inferring the properties of the corona from the
QPO, is that to date there is no self-consistent model that can fully explain the observed behaviour in a wide variety of sources.

In summary, current evidence - based on both imaging and timing techniques - strongly favours a compact corona located a few gravitational radii above the accretion disk. Whether the corona follows a slab, spherical, conical or patchy geometry, or whether it contains a purely thermal, nonthermal or hybrid population of electrons is still unknown. However, future emission models should ensure that the majority of the energy dissipation occurs in a compact region close to the central black hole, if they are to be physical. Of course, both X-ray macrolensing and soft-lag techniques are still in their early days, and the application of these techniques in determining the size and location of coronal regions should be checked against a much larger sample in the near future.

\section{ACKNOWLEDGEMENTS}

RCR is supported by NASA through the Einstein Fellowship Program, grant number PF1-120087. RCR thank Monica Valluri for the updated mass for NGC 4151 as well as Ed. Cackett and Dom Walton for helpful comments.

\section{REFERENCES}

Bentz M. C., Denney K. D., Cackett E. M., et al., 2006, ApJ, 651, 775

Berti E., Volonteri M., 2008, ApJ, 684, 822

Blackburne J. A., Pooley D., Rappaport S., 2006, ApJ, 640, 569

Cackett E. M., Fabian A. C., Zoghbi A., Kara E., Reynolds C., Uttley P., 2012, ArXiv e-prints

Chartas G., Kochanek C. S., Dai X., Poindexter S., Garmire G., 2009, ApJ, 693, 174

Dalal N., Kochanek C. S., 2002, ApJ, 572, 25

Dauser T., Garcia J., Wilms J., et al., 2013, ArXiv e-prints

De Marco B., Ponti G., Cappi M., et al., 2012, ArXiv e-prints

Doeleman S. S., Fish V. L., Schenck D. E., et al., 2012, Science, 338, 355

Elvis M., Maccacaro T., Wilson A. S., et al., 1978, MNRAS, 183, 129

Fabian A. C., Kara E., Walton D. J., et al., 2012, ArXiv e-prints

Fabian A. C., Zoghbi A., Ross R. R., et al., 2009, Nat, 459, 540

Falcke H., Biermann P. L., 1999, A\&A, 342, 49

Ghisellini G., Haardt F., Matt G., 2004, A\&A, 413, 535

Gierliński M., Middleton M., Ward M., Done C., 2008, Nat, 455, 369

Haardt F., Maraschi L., 1991, ApJ, 380, L51

Hirose S., Krolik J. H., De Villiers J.-P., Hawley J. F., 2004, ApJ, 606, 1083

Irwin M. J., Webster R. L., Hewett P. C., Corrigan R. T., Jedrzejewski R. I., 1989, AJ, 98, 1989

Johannsen T., Psaltis D., 2011, Phys. Rev. D, 83, 12, 124015

Kara E., Fabian A. C., Cackett E. M., Miniutti G., Uttley P., 2013, MNRAS, 430, 1408

McHardy I. M., Koerding E., Knigge C., Uttley P., Fender R. P., 2006, Nat, 444,730

Merloni A., Fabian A. C., 2001, MNRAS, 321, 549

Merloni A., Heinz S., di Matteo T., 2003, MNRAS, 345, 1057

Metcalf R. B., Zhao H., 2002, ApJ, 567, L5

Miller J. M., 2007, ARA\&A, 45, 441

Miller J. M., Miller M. C., Reynolds C. S., 2011, ApJ, 731, L5+

Miller L., Turner T. J., Reeves J. N., Braito V., 2010, MNRAS, 408, 1928

Miniutti G., Fabian A. C., 2004, MNRAS, 349, 1435
Morgan C. W., Kochanek C. S., Dai X., Morgan N. D., Falco E. E., 2008 , ApJ, 689, 755

Morgan C. W., Kochanek C. S., Morgan N. D., Falco E. E., 2010, ApJ, 712, 1129

Mortonson M. J., Schechter P. L., Wambsganss J., 2005, ApJ, 628, 594

Mosquera A. M., Kochanek C. S., Chen B., Dai X., Blackburne J. A. Chartas G., 2013, ArXiv e-prints

Narayan R., McClintock J. E., 2012, MNRAS, 419, L69

Narayan R., Yi I., 1994, ApJ, 428, L13

Onken C. A., Valluri M., Peterson B. M., et al., 2007, ApJ, 670, 105

Peng C. Y., Impey C. D., Rix H.-W., et al., 2006, ApJ, 649, 616

Pooley D., Blackburne J. A., Rappaport S., Schechter P. L., 2007, ApJ, 661, 19

Reis R. C., Miller J. M., Reynolds M. T., et al., 2012, Science, 337, 949

Reis R. C., Miller J. M., Reynolds M. T., et al., 2013, ApJ, 763, 48

Schnittman J. D., Krolik J. H., Noble S. C., 2012, ArXiv e-prints

Sluse D., Hutsemékers D., Courbin F., Meylan G., Wambsganss J., 2012, A\&A, 544, A62

Valluri M., Merritt D., Emsellem E., 2004, ApJ, 602, 66

Walton D. J., Nardini E., Fabian A. C., Gallo L. C., Reis R. C., 2013, MNRAS, 428, 2901

Wambsganss J., 2006, in Saas-Fee Advanced Course 33: Gravitational

Lensing: Strong, Weak and Micro, edited by G. Meylan, P. Jetzer,

P. North, P. Schneider, C. S. Kochanek, J. Wambsganss, 453-540

White N. E., Holt S. S., 1982, ApJ, 257, 318

Wilkins D. R., Fabian A. C., 2013, MNRAS, 602

Zdziarski A. A., Lubiński P., Smith D. A., 1999, MNRAS, 303, L11

Zhou X.-L., Wang J.-M., 2005, ApJ, 618, L83

Zhou X.-L., Zhang S.-N., Wang D.-X., Zhu L., 2010, ApJ, 710, 16

Zoghbi A., Fabian A. C., Reynolds C. S., Cackett E. M., 2012, MNRAS,

422,129 